\title{
Influence of Polymer Matrix Chemical Nature and Molding Technology on the Quality of Carbon Fiber Reinforced Polymer Parts
}

\author{
Tatiana Guzeva ${ }^{1}$, Maria Dronova ${ }^{1,}$, and Artyom Falman ${ }^{1}$ \\ ${ }^{1}$ Bauman Moscow State Technical University (BMSTU), 105005, Moscow, 5, 2nd Baumanskaya \\ Str., Russia
}

\begin{abstract}
The experimental research results of porosity, interlayer shear strength and flexural modulus of carbon fiber reinforced plastics (CFRP) depending on the chemical composition of the binder and molding technology are presented. The standard vacuum infusion technology and its two modifications differing in vacuum bag assembling order and initial material are applied. The best characteristics of CFRP are achieved with the improved technology №2 using two vacuum bags. The differences between two compounds with various hardener chemical nature are compared. Optimal characteristics are obtained by using compound with diaminodiphenylsulfone hardener.
\end{abstract}

\section{Introduction}

The primary quality markers for metal parts are machining accuracy and surface roughness. The analogy for polymer composite material (PCM) details is porosity $[1,2]$. The reason is in an impossibility of machining composites due to low interlayer shear strength therefore the delamination when processing can occur [3, 4]. Another feature of machining composites is intensive heat emission from cutting tools because of PCM's low thermal conductivity [5]. Cutting tools are facing intense wear since most reinforcing fibers possess abrasive properties. Thus, in the manufacture of PCM parts it is common to minimize mechanical processing and for a large range of parts, especially large-sized ones, processing is reduced to milling edges and making holes.

Polymer matrix chemical nature and molding technology have the greatest effect on PCM parts' porosity level. Nowadays majority of scientists generally research impact of only one composite material's component. The most common are characteristics and influence of polymer matrix [6-9] on any of quality parameters, e.g. strength. The issues related to the influence of the technology of forming parts from PCMs are considered less frequently [10-13]. Among the many technological parameters, the curing mode and its effect on the complex of mechanical properties are studied more thoroughly [10, 11].

The main goal of $[12,13]$ articles is in determining the impact of filler structure on the properties of the obtaining materials. However, the results are only suitable for macro-level

\footnotetext{
*Corresponding author: mariadv277@gmail.com
} 
and cannot be used when the analogical comparison for the micro- or nano-level is needed. The significant amount of articles is devoted to the technological mode, for example in articles [2, 3, 11] authors looked into different technological operations and tried to optimize technological parameters but results cannot be used widely.

The purpose of this article is the research of porosity and other PCM characteristics depending on the manufacturing technologies and compound properties.

\section{Objects and methods}

The research objects are two epoxy compounds based on epoxide resin ED-20, differing in hardener type:

- Triethylenetetramine (TETA), allowing room temperature curing;

- Diaminodiphenylsulfone (DDS), curing temperature $170^{\circ} \mathrm{C}$.

Unidirectional carbon tape FibArmTape-230/300 is used as reinforcement. Properties of the used materials are presented in table 1.

Table 1. Properties of the used materials

\begin{tabular}{|l|l|l|}
\hline \multirow{2}{*}{ Parameters } & Compound & DDS \\
\cline { 2 - 3 } & TETA & $2-3$ \\
\hline Curing shrink, \% & $3-4$ & $150-160$ \\
\hline Glass transition temperature, ${ }^{\circ} \mathrm{C}$ & $60-70$ & \\
\hline & Carbon tape \\
\hline Surface density, $\mathrm{g} / \mathrm{m}^{2}$ & 240 \\
\hline Flexural modulus, GPa & 250 & \\
\hline Elemental fiber strength, GPa & 3,5 & \\
\hline
\end{tabular}

The vacuum infusion technology was used as primal one in this research. The method consists in impregnating a dry material with a binder. Firstly, it is necessary to prepare the tooling surface and then lay out the fabric with the specified directions of reinforcement. In this work, as indicated above, an unidirectional tape was used. The evacuation of air from the vacuum bag is carried out using a pump.

The named technology has pros and cons which are needed to be considered during the research.

Advantages:

- Lesser porosity compared to hand molding;

- Lower equipment costs compared to resin transfer molding (RTM);

- Low fluctuation in mechanical properties;

- Cleaner production compared to hand molding;

- More resin-saving method compared to hand molding;

- Repeatability of composite properties from product to product.

Disadvantages:

- Large amount of disposable additional material (peel ply, resin-flow mesh, binder supply and pumping tubes, spiral tubes);

- High requirements for the qualification of the worker in order to obtain a product of satisfactory quality;

- The need for strict control during impregnation process.

Common vacuum infusion technology and two modifications were used for manufacturing test samples $[14,15]$. Technology No.1 utilized the special assembly with reusable elastic membrane and didn't require the new vacuum bag to be made every time when manufacturing samples. Technology No.2 used two vacuum bags. The first one was laid down on membrane, pumping out air with -0.7 bar pressure under surface. The 
drainage material was placed above and the second vacuum bag right after. The air was pumped out again, creating a pressure of -0.9 bar. Moreover, technologies differ by time needed for setting up vacuum bags (table 2). The set up time was determined during the manufacture of samples intended for interlayer shear testing. When accounting for the cost of additional materials, the cost of a reusable membrane was not considered. The qualification of the molder who made samples using the vacuum infusion technology was evaluated conditionally and divided into three groups: poor (in table 2 - "low"), medium and high.

Table 2. Characteristics of the vacuum infusion technologies used

\begin{tabular}{|l|l|l|l|}
\hline & \multirow{2}{*}{ Common technology } & \multicolumn{2}{|c|}{ Advanced technology } \\
\cline { 3 - 4 } & \multicolumn{1}{|c|}{1} & \multicolumn{1}{|c|}{ №1 } \\
\hline Vacuum bags & \multicolumn{1}{|c|}{1} & \multicolumn{1}{c|}{2} \\
\hline $\begin{array}{l}\text { Frequency of vacuum } \\
\text { bags usage }\end{array}$ & Single use & Reusable & Single use \\
\hline $\begin{array}{l}\text { Set up time for vacuum } \\
\text { bags, min }\end{array}$ & 15 & - & 30 \\
\hline $\begin{array}{l}\text { Cost of additional } \\
\text { materials, \% }\end{array}$ & 100 & 90 & 110 \\
\hline $\begin{array}{l}\text { Qualification of the } \\
\text { molder }\end{array}$ & Medium & Low & High \\
\hline
\end{tabular}

Technologies can be arranged in order of decreasing economic efficiency: advanced technology No.1, common technology, advanced technology No.2.

The porosity, flexural modulus and interlayer shear strength were measured in this research.

The porosity value was determined by hydrostatic weighing. The value of the adhesive strength was determined during tests for interlayer shear, using the short beam method GOST 32659-2014. The value of the flexural modulus was determined by thermomechanical analysis.

\section{Results and analysis}

Table 3 presents the average values of the CFRP characteristics. Five samples of each type were tested for evaluating porosity and interlayer shear stress and three samples for flexural modulus.

Table 3. CFRP properties depending on the molding technology

\begin{tabular}{|l|l|l|l|}
\hline \multirow{2}{*}{ Hardener chemical nature } & CFRP properties \\
\cline { 2 - 4 } & $\begin{array}{l}\text { Common vacuum } \\
\text { infusion technology }\end{array}$ & $\begin{array}{l}\text { Advanced vacuum infusion } \\
\text { technology }\end{array}$ \\
\cline { 3 - 4 } & & No.1 & No.2 \\
\hline \multicolumn{3}{|c|}{ Porosity, \% } \\
\hline Epoxy compound with TETA hardener & $3,1 \pm 0,2$ & $2,9 \pm 0,2$ & $2,2 \pm 0,2$ \\
\hline Epoxy compound with DDS hardener & $2,8 \pm 0,2$ & $2,1 \pm 0,2$ \\
\hline \multicolumn{3}{|c|}{ Interlayer shear stress, MPa } \\
\hline Epoxy compound with TETA hardener & $47 \pm 4$ & $46 \pm 4$ & $70 \pm 2$ \\
\hline Epoxy compound with DDS hardener & $67 \pm 2$ & $68 \pm 2$ & $79 \pm 5$ \\
\hline \multicolumn{3}{|l|}{ Modulus in bending, GPa } \\
\hline Epoxy compound with TETA hardener & $74 \pm 5$ & $76 \pm 5$ & $86 \pm 5$ \\
\hline Epoxy compound with DDS hardener & $77 \pm 5$ & $79 \pm 5$ & \\
\hline
\end{tabular}


According to the table 3, the properties for the same technology process are better using compound which cures at above room temperature (compound with DDS hardener, to be more specific).

Comparison of three molding technology demonstrates that the best characteristics of CFRP are achieved with the improved technology No.2 using two vacuum bags. Technology No.1 with elastic membrane gives results slightly better than common vacuum infusion. Therefore advanced vacuum infusion technologies allow to obtain CFRPs with higher mechanical characteristics, however the cost increases because of more expensive additional materials and vacuum bags set up duration.

Research analysis reveals that usage of epoxy compound with DDS hardener curing for several hours at $170^{\circ} \mathrm{C}$ in technology No.1 is impractical due to the need of heating the entire assembly. That requires large-volume drying ovens which is not economically rational. The utilization of this technology is also limited by the size and geometry of the molding parts. It is most suitable for flat samples like ones that are used in this study.

Thus, it is rational to compare only two technological processes: common vacuum infusion and advanced vacuum infusion technology No.2 with two vacuum bags. The use of improved technology reduced porosity by approximately $30 \%$ and increased interlayer shear strength and flexural modulus by about $6 \%$. All the named advantages are achieved using the room temperature curing compound (with TETA hardener). Utilization of DDS hardener gave analogical results decreasing porosity by $25 \%$ and increasing interlayer shear strength and flexural modulus by $4 \%$ and $11 \%$ accordingly.

The compound composition has a significant impact on all the characteristics of CFRP and they are lower while using TETA hardener compared to DDS hardener. For instance, interlayer shear strength when using advanced technology No.2 is $42 \%$ lower and the porosity on the contrary is $5 \%$ higher. Thus, compounds which cure above room temperature applicated in any of molding technologies can provide obtaining PCMs with superior mechanical characteristics comparing with the room temperature curing ones.

Varying the molding technology has rather poor influence on CFRP characteristics. The flexural modulus changes by $4,2 \%$ and $4,5 \%$ when using compounds with TETA and DDS hardeners accordingly.

\section{Conclusions}

Comparison of three molding technology proves that the best characteristics of CFRP are achieved with the improved technology No.2 using two vacuum bags. It reduced porosity by $30 \%$ and rised interlayer shear strength and flexural modulus by $6 \%$. These advantages were obtained with room temperature curing compound with TETA hardener. Changing hardener to DDS gives the similar results in decreasing porosity by $25 \%$ and increasing interlayer shear strength and flexural modulus by $4 \%$ and $11 \%$ accordingly. Thus, if the best mechanical characteristics are required, the advanced technology No.2 and higher curing temperature compound are needed.

The molding technology No.1 with the elastic membrane demonstrates better results than common infusion technology but significantly uprises cost due to the necessity of large-volume drying ovens for compounds which require high temperatures for curing.

\section{References}

1. Rudskoi A.I., Baurova N.I. Technological heredity during the production and operation of structural materials. Russian Metallurgy, v. 13. pp. 1378-1383. (2019). 
2. Gorodetskii M.A., Nelyub V.A., Malysheva G.V., Shaulov A.Y., Berlin A.A. Technology of forming and the properties of reinforced composites based on an inorganic binder. Russian Metallurgy, v. 13. pp.1195-1198. (2018).

3. Baurova N.I., Zorin V.A. Technological heredity in the production of machines from polymer composite materials: monograph. - M. MADI. 220 p. (2018).

4. Kobets L.P., Borodulin A.S., Malysheva G.V. Study of microcapillary impregnation of carbon fiber by epoxy binder. Fibre Chemistry, v. 48 (4). pp. 311-315. (2016).

5. Galinovsky A.L., Bochkarev S.V., Nelyub V.A. Technologies for the production and diagnostics of composite structures of aircraft. Study guide, Stary Oskol. 332 p. (2019).

6. Malysheva G.V., Akhmetova E.Sh., Marycheva A.N. Estimation of glass transition temperature of polysulfone-modified epoxy binders. Glass Physical and Chemistry. v.40 (5). pp.543-548. (2014).

7. Borodulin A.S., Marycheva A.N., Malysheva G.V. Simulation of impregnation kinetics of fabric fillers in the production of fiberglass articles. Glass Physics and Chemistry, v. 41(6). pp. 660-664. (2015).

8. Malysheva G.V., Shablygin M.V., Guzeva T.A. Method for assessing aramide fiber microstructure features. Fibre Chemistry, v. 47 (2). pp. 85-88. (2015).

9. Baurova N., Anoprienko A., Romanova Y. Providing dismountable rivet bonded joints through the use of hot-melt joints through the use of hot-melt adhesives ICMTMTE T. 129. (2017).

10. Kosenko E.A., Baurova N.I., Zorin V.A. Service properties of composites with various types of hybrid matrices. Russian Metallurgy (Metally), v. 13, pp. 1526-1530. (2020).

11. Maung P.P., Malysheva G. Modeling the effect of fabric weaving pattern om the impregnation process in the manufacturing of the fiber reinforced plastic structures. AIP Conference proceeding. v.2171. (2019).

12. Baurova N.I., Zorin V.A., Prikhodko V.M. Determination of the synergetic effect of the damage accumulation process in polymer materials using catastrophe theory. Theoretical Foundations of Chemical Engineering, v. 50 (1). pp.119-125. (2016).

13. Malysheva G.V., Kirsanova E.A., Mauang P.P., Tumasova M.S. Rigidity of glass carbon and organic yarns of special-purpose fabrics. Fibre Chemistry. v.48 (2). pp. 140-142. (2016).

14. Chudnov I.V., Nelyub V.A., Marycheva A.N. Molding parts from carbon plastics using vacuum infusion technology using a reusable elastic membrane. All Materials. Encyclopedic reference book. №. 11. pp.43-47. (2019)

15. Nelyub V.A. Improving the quality of molding carbon plastics based on epoxy binders and metallized reinforcing materials using vacuum infusion technology. Glue.

Sealants. Technologies. №. 9. pp.40-43. (2020). 